\title{
Patrones de consumo de bebidas alcohólicas en fiestas juveniles que dependen de diferencias individuales y personalidad
}

\author{
Patterns of alcoholic consumption in youth's parties asociated to individual \\ differences and personality
}

\author{
Christian Enrique Cruz Torres* \\ Carlos Cesar Contreras Ibáñez * \\ Universidad de Guanajuato, Campus León* \\ Universidad Autónoma Metropolitana, Unidad Iztapalapa**
}

\section{RESUMEN}

Se analiza la hipótesis de que jóvenes con diferentes niveles de depresión, ansiedad, sociabilidad, búsqueda de sensaciones y autoconciencia presenten diferentes patrones de consumo de bebidas alcohólicas en fiestas como una estrategia para enfrentar demandas de socialización. Participaron 640 jóvenes, 51\% mujeres, con edad promedio de 17 años. Se diseñaron instrumentos en formato tipo Likert de autorreporte para medir las diferencias individuales y la frecuencia de síntomas de intoxicación alcohólica en fiestas. Los resultados muestran que la depresión se asocia con una mayor frecuencia de consumo hasta alcanzar síntomas de intoxicación alta (náuseas y pérdida de la conciencia); ansiedad y búsqueda de sensaciones se relacionan con una mayor frecuencia en síntomas de intoxicación intermedia (dificultades para hablar); autoconciencia y sociabilidad no presentan asociaciones significativas. Los resultados respaldan la hipótesis de que el consumo de alcohol es un recurso para hacer frente a niveles altos de depresión (beber hasta perder la conciencia, como una forma de evadir la realidad), ansiedad (consumo medio, para reducir el estrés de interacciones sociales), y búsqueda de sensaciones (consumo moderado, asociado a euforia).

Palabras clave: socialización, consumo de alcohol, jóvenes, depresión, ansiedad.

\section{ABSTRACT}

An analysis of the hypothesis that young people with varying degrees of depression, anxiety, sociability, thrill seeking behavior, and self-awareness have different patterns of consumption of alcoholic beverages at parties as a strategy to cope with the demands of socializing. In this study, 640 young people took part (51\% women, with an average age of 17). Self-administered Likert-type instruments were designed to measure the individual differences and the frequency of the symptoms of drunkenness at parties. The results show that depression is most frequently linked to consumption leading to high-levels of intoxication (nausea and loss of consciousness); anxiety and thrill seeking are associated with a greater frequency of medium-level intoxication (difficulties in speaking); self-awareness and sociability do not present statistically significant associations. These results support the hypothesis of alcohol consumption as a means of facing high levels of depressions (drinking until falling unconscious, as a way of avoiding reality), anxiety (medium-level consumption, in order to reduce the stress of social interactions), and the thrill seeking (moderate consumption, linked to euphoria).

Keywords: socialization, alcohol consumption, young people, depression, anxiety.

Fecha de recepción: 1 de febrero de 2015 Fecha de aceptación: 2 de junio de 2015

Para correspondencia: Christian Enrique Cruz Torres, cassiel.79@gmail.com 
El consumo excesivo de alcohol es un grave problema de salud pública, asociado a enfermedades hepáticas, cardiovasculares y cerebro-vasculares, ubicadas entre las principales causas de muerte en México (Secretaría de Salud, 2005). Además, es una causa importante de accidentes automovilísticos y lesiones debidas a actos violentos que requieren atención hospitalaria de urgencias (Casanova, Borges, Mondragón, MedinaMora \& Cherpitel, 2001; Orozco, Borges, Mondragón \& Monroy-Nasr, 2005).

Diferentes mediciones identifican un incremento en los índices de consumo entre adolescentes. En 2008, $35.7 \%$ de los varones y $27.7 \%$ de las mujeres adolescentes (12 a 17 años) declararon consumir alcohol en alguna medida, se incrementó este indicador a $46 \%$ para los hombres y 39.7\% para las mujeres en 2011 (Medina-Mora et al., 2012).

Este crecimiento en el consumo entre adolescentes resulta más preocupante al considerar estudios que identifican esta etapa como crucial en la formación y la modificación de patrones de consumo de bebidas alcohólicas. La Encuesta de Consumo de Drogas en Estudiantes del Distrito Federal reporta que en 2000, $22 \%$ de los estudiantes de secundaria declaró haber consumido bebidas alcohólicas en el mes previo a la encuesta, porcentaje que se incrementó a $43 \%$ para los estudiantes de nivel medio superior (Villatoro et al., 2001). Este salto continúa en la medición del (Villatoro et al., 2004), observándose además un incremento en el consumo en ambos segmentos (24\% en secundaria y $51 \%$ en bachillerato). La medición de 2006 (Villatoro et al., 2007) reporta el mismo patrón, e identifica el paso de la escuela secundaria (29\%) al nivel medio superior (54\%) como un parteaguas en los patrones de consumo de bebidas alcohólicas. Este mismo seguimiento en 2012 muestra un porcentaje menor en los estudiantes de secundaria (27.2\%), pero más alto para los estudiantes de nivel medio superior (56.5\%) (Villatoro et. al., 2013).

Con respecto al abuso en el consumo, la medición de 2003 (Villatoro et al., 2004) señala que $15.5 \%$ de los jóvenes que cursan la secundaria reportan haber abusado del alcohol en el mes de aplicación; esta cifra se incrementa a $36.3 \%$ entre los estudiantes de bachillerato y a $34.9 \%$ en escuelas técnicas de nivel medio superior. Este indicador disminuye, pero conserva las diferencias entre los niveles educativos en la medición de 2012, con 13.5\% para secundaria y 33.9\% para bachillerato (Villatoro et al., 2013). Estudios previos muestran que este cambio en los patrones de consumo tiende a continuarse hasta la edad adulta, resaltando la importancia de estudiar los factores de riesgo del consumo excesivo de alcohol en esta etapa de la vida (McCambridge, Mc Alaney \& Rowe, 2011).

En psicología social se han realizado esfuerzos por explicar el consumo excesivo de alcohol. Se ha observado que la ansiedad social y los desórdenes ligados a la misma (por ejemplo, temor a la evaluación social negativa) se asocian con un mayor consumo de alcohol en personas adultas (Ham, Debra \& Hope, 2003; Ham, Hope, White \& Rivers, 2002). Otro trastorno ligado al consumo de alcohol es la depresión, el consumo excesivo se relaciona con mayores índices de depresión (Lukassen \& Beaudet, 2005; Echeburúa, 2001). Estas investigaciones midieron la ansiedad y la depresión como trastornos de personalidad. Retomar para este estudio la misma perspectiva teórica implicaría que los resultados obtenidos sólo podrían generalizarse hacia una población con trastornos de la personalidad clínicamente diagnosticados.

Si se enfoca en rasgos de la personalidad y no en sus trastornos, la ansiedad y la depresión son entendidas como diferencias individuales que forman parte, en alguna medida, de la personalidad de cualquier individuo (McCrae \& Costa, 1990, 1997; Costa \& Widieger, 1994). Desde uno de estos enfoques, las características de personalidad son clasificadas en cinco grandes rasgos (neuroticismo, extroversión, apertura, autoconciencia y agradabilidad), que son "dimensiones perdurables de diferencias individuales que tienden a mostrarse en patrones consistentes de pensamientos, sentimientos y acciones" (McCrae \& Costa, 1990, p. 23). La ansiedad y la depresión son concebidas como dos de las diferencias individuales que forman parte del neuroticismo (para una revisión de otros enfoques de personalidad vid. Funder, 2001).

Esta concepción implica que, aunque todos podemos presentar episodios ocasionales de ansiedad o depresión, hay quienes los padecen con mayor frecuencia sin que necesariamente crucen el umbral que caracterizaría a un trastorno de la personalidad. En consecuencia, en las personas pueden evaluarse niveles de estas diferencias individuales. 
Aquellos con altos índices de ansiedad son aprehensivos, temerosos, con tendencia a la preocupación, y nerviosos; mientras que quienes presentan índices bajos son más calmados, relajados y no les preocupa tanto que las cosas puedan salir mal. Por su parte, quienes reportan altos grados de depresión tienden a experimentar, con mayor frecuencia, sentimientos de culpa, tristeza, desesperanza, y soledad (Costa \& Widieger, 1994).

Una tercera diferencia individual asociada al consumo de bebidas alcohólicas es la búsqueda de sensaciones, una necesidad disposicional por altos niveles de estimulación. Estudios previos han mostrado que niveles altos en esta diferencia individual se asocian con un mayor uso (Martin et al., 2002) y abuso (Johnson \& Cropsey, 2000) de bebidas alcohólicas en la adolescencia, y una mayor participación en juegos organizados en fiestas para incrementar el consumo de bebidas alcohólicas (Diulio, Silvestri \& Correia, 2014). Por último, la diferencia individual conocida como autoconciencia, que define a personas que se avergüenzan con facilidad, se sienten incómodas rodeadas de gente y son particularmente sensibles al ridículo (Costa \& Widieger, 1994), se ha asociado a un consumo más elevado (Hull \& Young, 1983; Pluddermann, Theron \& Steel, 1999).

Las investigaciones citadas muestran que quienes reportan índices más altos de ansiedad, depresión, autoconciencia y búsqueda de sensaciones reportan también un uso más frecuente o excesivo de bebidas alcohólicas. Sin embargo, para entender esos patrones es necesario considerar que la ingesta de bebidas alcohólicas está motivada por la búsqueda de los efectos psicofisiológicos producto de su intoxicación. Investigaciones previas en jóvenes (Camacho, 2005) han mostrado que el consumo de bebidas alcohólicas está asociado a expectativas tan diversas como, por ejemplo, que sus efectos faciliten la interacción con otras personas, mejoren la capacidad para expresarse verbalmente, les permitan desinhibirse, incrementen la agresividad y la sexualidad, entre otras. Esta diversidad de expectativas tiene sentido, puesto que los efectos de la intoxicación alcohólica sobre la conducta, las emociones y el pensamiento varían de acuerdo con su intensidad (Dubowsky, 1977).
La búsqueda de diferentes efectos psicofisiológicos podría llevar a patrones diferenciados de consumo de alcohol, donde los niveles de ingesta se asocian a problemas particulares, que resultan de los altos niveles de ciertas diferencias individuales. De esta forma, quienes reporten índices altos en ansiedad y autoconciencia consumirían alcohol buscando la desinhibición, el relajamiento y la euforia, característicos de las primeras etapas de la intoxicación alcohólica como un recurso para enfrentar las situaciones potencialmente estresantes propias de una reunión social. Por su parte, quienes reporten índices altos en depresión podrían alcanzar con mayor regularidad los niveles más altos de intoxicación (pérdida de la conciencia) como una vía de escape a la realidad.

Otra diferencia individual que podría asociarse, aunque de manera indirecta, al consumo de alcohol es la sociabilidad. Ésta es definida como la preferencia por la compañía de otras personas. Las personas con índices altos en este indicador tienden a disfrutar mucho la compañía de otros, mientras más personas mejor. Quienes reportan puntajes bajos tienden a ser solitarios, e incluso evitan activamente la estimulación social (Costa \& Widieger, 1994). Estudios previos (Pulido et al., 2002) reportan que los lugares de mayor consumo son las discotecas o bares. Es necesario explorar la posibilidad de que quienes disfrutan de sitios donde se reúne mucha gente asistan más a menudo a fiestas juveniles en general, teniendo mayor acceso a las bebidas alcohólicas que comúnmente se consumen en estos lugares. Esto implicaría un factor de riesgo para su consumo excesivo.

Los reportes epidemiológicos muestran un incremento en el consumo entre los adolescentes, y las investigaciones adicionales identifican este periodo como determinante en el establecimiento de hábitos de consumo para el resto de la vida. Estudios sobre la personalidad muestran que la ansiedad, la depresión, la búsqueda de sensaciones, la autoconciencia y la sociabilidad están asociadas a un mayor consumo. Considerando esos antecedentes, este estudio tiene por objetivo explorar si estas diferencias individuales generan patrones diferenciados de consumo de bebidas alcohólicas en fiestas. Se asume que estas diferencias resultarían de una búsqueda de diferentes niveles de intoxicación para hacer frente a las limitaciones 
personales y demandas características de las interacciones sociales en las fiestas.

\section{MÉTODO}

\section{Participantes}

Participaron 640 estudiantes de dos colegios de bachilleres de la Ciudad de México; 51\% fue mujer, la edad media fue de 17 años con una desviación estándar de 2.2; $48 \%$ se encontraba en los primeros dos semestres del bachillerato; $59 \%$ pertenecía al turno matutino; 66\% reportó no trabajar, 29\% trabajaba para cubrir algunos gastos y $5 \%$ trabajaba para sostenerse económicamente. El promedio académico reportado fue de 7.7.

\section{Instrumentos}

El cuestionario fue de autoaplicación. En la primera sección se encontraban las mediciones de diferencias individuales de depresión (siete reactivos), ansiedad (siete reactivos), búsqueda de sensaciones (cuatro reactivos), autoconciencia (cuatro reactivos) y sociabilidad (cinco reactivos), diseñadas ex profeso para esta investigación con base en las definiciones teóricas (Costa \& Widieger, 1994). Se presentaron en formato tipo Likert con opciones de respuesta que iban de 1 (totalmente en desacuerdo) a 6 (totalmente de acuerdo), se suprimió el punto medio para prevenir que los participantes lo eligieran de manera consistente por constituir una respuesta menos comprometida (Albarracín, Blair, Mark \& Zanna, 2005).

Enseguida, en el cuestionario se ubicaron reactivos destinados a medir los índices de consumo de bebidas alcohólicas con base en los síntomas reportados de intoxicación, diseñados ex profeso para este estudio. Estos cuatro reactivos medían la cantidad de ocasiones en las que los y las jóvenes alcanzaban síntomas particulares de intoxicación por alcohol: dificultad para hablar, mareos, náuseas y pérdida de la conciencia (por ejemplo, ¿En cuántas de las ocasiones que asististe a una fiesta o un antro en lo que va del año ingeriste bebidas alcohólicas hasta que te sentiste con náuseas?). Además, se preguntó la cantidad de fiestas a las que habían asistido en el año en curso hasta el momento de la encuesta. Se midió el consumo de bebidas alcohólicas mediante sus síntomas de intoxicación y no por número de copas por ocasión de consumo durante el último mes, semana o año al considerar que el nivel de intoxicación alcohólica está en función de factores adicionales a la cantidad de copas ingeridas, como la talla y el sexo. Suponemos, además, que la mayoría de las personas tienden a regular su consumo con base en los síntomas de intoxicación que van experimentando y no en la cantidad de copas ingeridas.

\section{Procedimiento}

El muestreo fue intencional, no probabilístico, por conveniencia; en la muestra se incluyeron a aquellos alumnos cuyos profesores nos brindaron un tiempo de su clase para la aplicación de inventarios. Una vez obtenido el consentimiento informado de los participantes se procedió a la aplicación de los cuestionarios dentro de las aulas.

\section{RESULTADOS}

\section{Validación y estructura de las mediciones de diferencias individuales}

Para obtener evidencias de validez de constructo de los instrumentos de diferencias individuales se utilizaron análisis factoriales de componentes principales con rotación ortogonal Varimax y normalización de Kayser para cada una de las mediciones por separado. Todos los análisis produjeron soluciones unifactoriales. Excepto el instrumento de sociabilidad, de donde se retiraron dos reactivos por no agregarse al factor principal, todos los instrumentos conservaron sus reactivos originales. La consistencia interna de cada instrumento se calculó mediante la fórmula Alfa de Cronbach (vid. tabla 1). Posteriormente, se formaron nuevas variables sumando los reactivos correspondientes a cada escala.

Tomando como criterio de división la media de cada variable se formaron grupos altos y bajos en cada una de las diferencias individuales. Las pruebas $t$ de Student muestran que el criterio fue adecuado, y se obtuvieron diferencias estadísticamente significativas entre los grupos para cada variable (todas las $t>20$, significativas a $<.01$ ).

Debe considerarse que las diferencias individuales no se excluyen entre sí, y coexisten a menudo en la personalidad de un mismo individuo. Para obtener un 
marco interpretativo a este respecto utilizamos correlaciones Pearson entre los índices de cada diferencia individual (vid. tabla 2).

\section{Patrones de consumo de bebidas alcohólicas}

Con base en un estudio previo (Pulido et al., 2002), se seleccionaron las fiestas juveniles para medir el consumo de bebidas alcohólicas. Se utilizaron correlaciones Pearson entre la variable de asistencia a fiestas y cada uno de los indicadores de consumo para verificar que este contexto fuera correcto.

Asistir con asiduidad a fiestas se relaciona con una mayor frecuencia de consumo de alcohol hasta marearse $(\mathrm{r}=.727)$, presentar dificultades para hablar $(\mathrm{r}=.505)$, sentir náuseas $(\mathrm{r}=.407)$ y perder la conciencia $(r=.233)$, todas significativas con $\mathrm{p}<.001$. El hecho de que la asociación sea menor para los síntomas que indican un consumo más alto por ocasión puede señalar que una mayor frecuencia de consumo moderado se debe, en buena medida, al simple acceso a las bebidas alcohólicas en fiestas. Sin embargo, los síntomas de intoxicación asociados a un consumo más intenso reciben una mayor influencia de otros factores.

Estas correlaciones muestran que mayor asistencia a fiestas implica mayor frecuencia aunque no necesariamente un patrón más fuerte de consumo; quien reporta asistir a 50 fiestas y haber bebido hasta marearse en diez de ellas presenta un patrón de consumo diferente a quien asiste a sólo a diez fiestas y declara haber consumido alcohol hasta marearse en las diez.

Para controlar este efecto se calculó un porcentaje para cada indicador de consumo tomando en cuenta la cantidad de fiestas a las que asistió. Así, el primer participante del ejemplo anterior habría consumido bebidas alcohólicas hasta marearse en $20 \%$ de las fiestas, mientras que el segundo tendría un índice de 100\%.

Tabla 1. Indicadores de las escalas de diferencias individuales

\begin{tabular}{|c|c|c|c|c|}
\hline Factores & $\begin{array}{l}\text { Varianza } \\
\text { explicada }\end{array}$ & Confiabilidad & Media & Rango \\
\hline Depresión (7 reactivos) & $44.7 \%$ & 0.78 & 15.7 & Mín.: 7 \\
\hline A menudo me siento deprimido & & & & Máx.: 43 \\
\hline \multicolumn{5}{|l|}{$\begin{array}{l}\text { Frecuentemente siento que mi vida no tiene } \\
\text { solución }\end{array}$} \\
\hline Ansiedad (7 reactivos) & $35.3 \%$ & 0.72 & 19 & Mín.: 7 \\
\hline Frecuentemente me siento ansioso & & & & Máx.: 43 \\
\hline \multicolumn{5}{|l|}{$\begin{array}{l}\text { A menudo me siento tenso por las cosas que } \\
\text { pasan a mi alrededor }\end{array}$} \\
\hline Búsqueda de sensaciones (4 reactivos) & $47.4 \%$ & 0.62 & 15.8 & Mín.: 4 \\
\hline Disfruto arriesgarme a probar cosas nuevas & & & & Máx.: 24 \\
\hline \multicolumn{5}{|l|}{$\begin{array}{l}\text { Frecuentemente estoy en busca de emociones } \\
\text { fuertes }\end{array}$} \\
\hline Autoconciencia (4 reactivos) & $43.3 \%$ & 0.56 & 11.8 & Mín.: 4 \\
\hline $\begin{array}{l}\text { Cuando estoy con gente me angustia hacer el } \\
\text { ridículo }\end{array}$ & & & & Máx.: 24 \\
\hline \multicolumn{5}{|l|}{ A menudo me siento inferior a quienes me rodean } \\
\hline Sociabilidad (3 reactivos) & $50.5 \%$ & 0.5 & 12.5 & Mín.: 3 \\
\hline Disfruto más estar en compañía de otros que solo & & & & Máx.: 18 \\
\hline $\begin{array}{l}\text { Me gusta estar en lugares donde se reúne mucha } \\
\text { gente }\end{array}$ & & & & \\
\hline
\end{tabular}


Tabla 2. Correlaciones Pearson entre escalas de diferencias individuales

\begin{tabular}{l|c|c|c|c|c} 
& Sociabilidad & Autoconciencia & $\begin{array}{c}\text { Búsqueda de } \\
\text { sensaciones }\end{array}$ & Depresión & Ansiedad \\
\hline Sociabilidad & 1 & & & & \\
\hline Autoconciencia & 0.063 & 1 & & & \\
\hline $\begin{array}{l}\text { Búsqueda de } \\
\text { sensaciones }\end{array}$ & $.570^{* *}$ & -0.029 & 1 & & \\
\hline Depresión & -0.021 & $.548^{* *}$ & -0.064 & 1 & \\
\hline Ansiedad & 0.042 & $.524^{* *}$ & 0.072 & $.625^{* *}$ & 1 \\
\hline
\end{tabular}

${ }^{*} \mathrm{p}=<.001$.

Tabla 3. Índices de consumo con base en los síntomas de intoxicación reportados

\begin{tabular}{c|c|c|c|c|c|c|c|c}
\multicolumn{3}{c|}{ Hombres } & \multicolumn{2}{c}{ Mujeres } \\
\hline & $\begin{array}{c}\text { No } \\
\text { recordar } \\
\text { el día } \\
\text { anterior } \\
(\%)\end{array}$ & $\begin{array}{c}\text { Náuseas } \\
(\%)\end{array}$ & $\begin{array}{c}\text { Dificultad } \\
\text { para hablar } \\
(\%)\end{array}$ & Mareo(\%) & $\begin{array}{c}\text { No } \\
\text { recordar el } \\
\text { día anterior } \\
(\%)\end{array}$ & $\begin{array}{c}\text { Náuseas } \\
(\%)\end{array}$ & $\begin{array}{c}\text { Dificultad } \\
\text { para hablar } \\
(\%)\end{array}$ & $\begin{array}{c}\text { Mareo } \\
(\%)\end{array}$ \\
\hline $0 \%$ & 79.0 & 64.8 & 58.8 & 37.2 & 89.5 & 64.8 & 71.3 & 41.9 \\
\hline $\begin{array}{c}\text { De 1\% a } \\
30 \%\end{array}$ & 16.0 & 23.3 & 21.7 & 18.4 & 8.9 & 23.3 & 17.2 & 20.8 \\
\hline $\begin{array}{c}\text { De 31 a } \\
60 \%\end{array}$ & 1.6 & 6.4 & 9.7 & 22.0 & 1.2 & 6.4 & 9.0 & 20.7 \\
\hline $\begin{array}{c}\text { De 61\% } \\
\text { a 100\% }\end{array}$ & 3.4 & 5.5 & 8.8 & 20.6 & 0.8 & 5.5 & 2.5 & 2.5 \\
\hline
\end{tabular}

Puntajes altos en este indicador no implican una alta frecuencia de consumo, sino un mayor abuso de la ingesta de bebidas alcohólicas cuando están disponibles. La tabla 3 muestra los grupos porcentuales para cada uno de los síntomas en hombres y mujeres.

Aunque los patrones de consumo son similares para hombres y mujeres, las diferencias se hacen notar en los indicadores de mayor consumo. Por ejemplo, $0.8 \%$ de las mujeres declaró haber bebido hasta no recordar lo que hizo el día anterior entre $61 \%$ y $100 \%$ de las veces que asistió a una fiesta, mientras que los hombres reportan $3.4 \%$.

Para analizar posibles relaciones entre las diferencias individuales y los patrones de consumo se utilizaron análisis de varianza factoriales para cada diferencia individual, teniendo como variables dependientes los indicadores de consumo basados en los síntomas de intoxicación. Para probar los efectos principales se utilizaron análisis de varianza simples.
La figura 1 muestra los porcentajes para hombres y mujeres altos y bajos en depresión en cada síntoma de intoxicación. No se encontraron efectos principales ni interacciones significativas para el indicador de consumir alcohol hasta marearse (F's $<1.5)$.

En el caso de consumir alcohol hasta presentar dificultades para hablar se observó sólo el efecto principal del sexo de los participantes; los hombres (16.9\%) reportaron porcentajes de intoxicación más altos que las mujeres (9.1\%) F (1468) = 9.7, p = .002.

$\mathrm{El}$ análisis del consumo hasta sentir náuseas refleja sólo el efecto principal de depresión y un efecto marginalmente significativo del sexo. Los hombres (11.6\%) alcanzan este nivel de intoxicación más a menudo que las mujeres $(7.5 \%) \mathrm{F}(1484)=3.7, \mathrm{p}=.053$; $\mathrm{y}$ los participantes con mayor depresión (12.7\%) llegan a este nivel de intoxicación con más frecuencia que quienes reportaron niveles bajos (6.9\%) F (1478) = 7.5, p = .006. 
El indicador de pérdida de conciencia muestra efectos principales de los niveles de depresión y el sexo de los participantes sin interacciones entre ellas. Los hombres (6.0\%) reportan este síntoma con más frecuencia que las mujeres $(2.2 \%) \mathrm{F}(1493)=6.7$, $\mathrm{p}=.010$. Quienes presentan niveles altos de depresión (6.3\%) experimentan este síntoma más frecuentemente que quienes tienen niveles bajos (2.3\%) F (1485) = 7.0, $\mathrm{p}=.008$.

Posteriormente se analizó la ansiedad (vid. figura 2). Para el indicador de presentar mareos se observó un efecto principal marginalmente significativo $\mathrm{F}(1457)=3.3, \mathrm{p}=.06)$. Quienes reportaron índices altos de ansiedad (34.0\%) declararon consumir alcohol hasta marearse con mayor frecuencia que quienes reportaron índices menores (27.5\%).
En el indicador de dificultades para hablar se observan sólo efectos principales del sexo y la ansiedad. Los hombres (16.9\%) presentaron este síntoma con más frecuencia que las mujeres (9.0\%) F (1468) = 9.75, $\mathrm{p}=.002$. Quienes reportaron puntajes altos de ansiedad experimentaron este síntoma con más frecuencia (17.1\%) que quienes mostraron puntajes bajos (8.7\%) $\mathrm{F}(1462)=10.9, \mathrm{p}=.001$.

El análisis del indicador de experimentar náuseas presenta un efecto principal debido a la ansiedad. Quienes reportaron niveles altos de ansiedad alcanzaron con mayor frecuencia este síntoma $(27.0 \%)$ que quienes presentaron niveles bajos (18.8\%) F (1479) = $6.4, \mathrm{p}=.012$.

Los análisis para el indicador de pérdida de la conciencia arrojan efectos principales asociados al sexo

\section{Figura 1. Porcentajes de síntomas de intoxicación para grupos bajos y altos en depresión}

Porcentaje de

síntomas

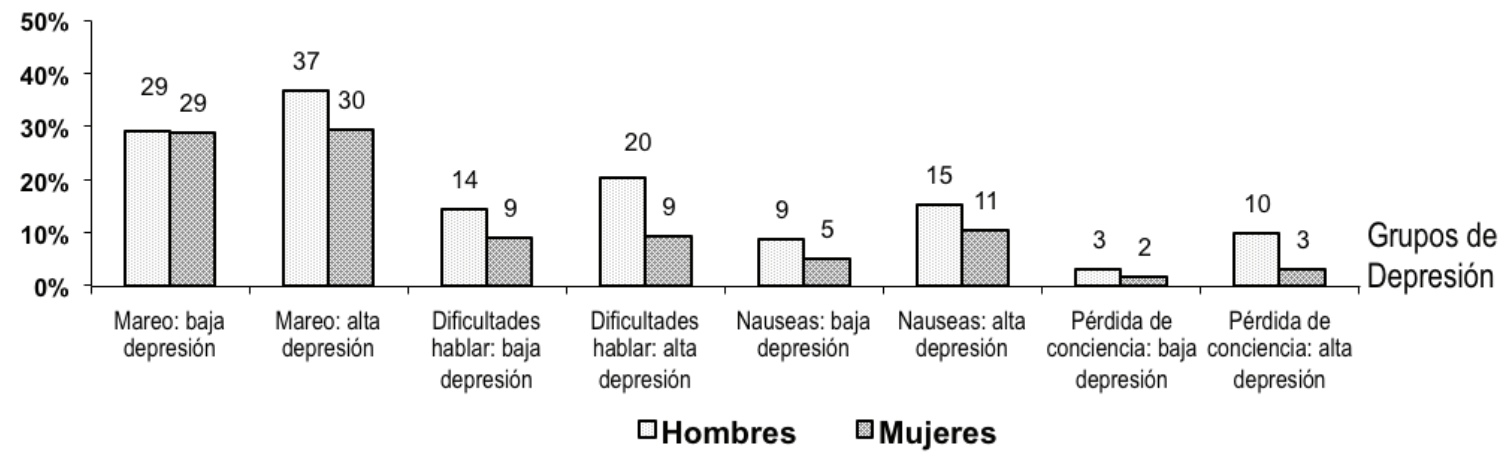

Figura 2. Porcentajes de síntomas de intoxicación para grupos bajos y altos en ansiedad

Porcentaje de

sintomas

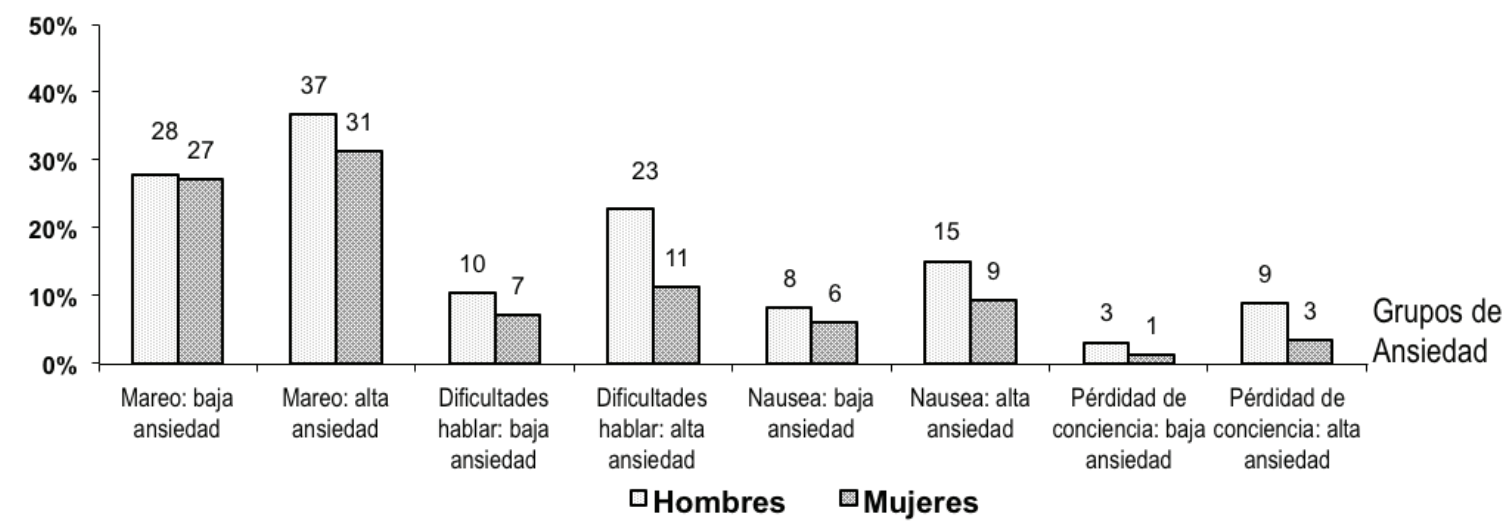


de los participantes (ya reportado) y sus niveles de ansiedad. Quienes reportaron niveles altos de ansiedad presentaron una mayor tendencia a consumir hasta perder la conciencia (6.2\%) que quienes reportaron puntajes bajos (2.1\%) F (1486) $=7.8, \mathrm{p}=.005$.

Los índices de sociabilidad no produjeron efectos estadísticamente significativos sobre las tendencias a reportar los diferentes síntomas de intoxicación (todas las $\mathrm{F}<1$ ). Esto indicaría que una alta necesidad de convivencia social no implica, necesariamente, asistir con mayor regularidad a fiestas u otros sitios de reunión donde se tiene acceso a bebidas alcohólicas. Una correlación de Pearson no significativa entre ambos indicadores confirma esta hipótesis $(\mathrm{r}=.037, \mathrm{p}=.385)$.

La diferencia individual de búsqueda de sensaciones sólo muestra efectos sobre el indicador de beber alcohol hasta presentar dificultades para hablar. Los hombres reportaron una mayor tendencia que las mujeres por ingerir alcohol hasta alcanzar este síntoma (ya reportado). Además, con independencia del sexo, quienes presentaron puntajes altos en ansiedad mostraron también una mayor proporción en este síntoma (15.8\%) que quienes reportaron puntajes bajos (9.4\%) $\mathrm{F}(1462)=6.3, \mathrm{p}=.012$.

Finalmente, las variaciones en los niveles de autoconciencia no produjeron efectos sobre ninguno de los indicadores de consumo (todas las $\mathrm{F}<1$ ).

\section{DISCUSIÓN}

En congruencia con nuestra hipótesis general, cada diferencia individual se asoció a distintas tendencias de consumo. Esto indicaría que niveles altos en cada diferencia individual implican problemáticas distintas que motivan al individuo a buscar efectos específicos de la intoxicación para hacerles frente.

El análisis del indicador de depresión muestra que los participantes con índices altos de esta diferencia individual tienden, con mayor regularidad, a consumir bebidas alcohólicas hasta alcanzar los síntomas más extremos de intoxicación (náuseas y pérdida de la conciencia); diferencias que no se observaron en los indicios de intoxicación menor (mareos y dificultad para hablar). Si consideramos que estas personas más a menudo tienden a experimentar culpa, tristeza, desesperanza, y soledad (Costa \& Widieger, 1994), es posible que las fiestas, el consumo de alcohol y los síntomas de intoxicación severa se conviertan en un escape de las emociones negativas que reiteradamente les produce una realidad que los sobrepasa y donde no perciben ninguna esperanza de cambio positivo.

El análisis de los niveles de ansiedad muestra que cuando los participantes con puntajes altos de ésta consumen bebidas alcohólicas, alcanzan con mayor regularidad síntomas de intoxicación alcohólica de moderada a grave. A diferencia de la depresión, estas distinciones se acentúan para el indicador de consumir bebidas alcohólicas hasta presentar dificultades para hablar, un indicador de intoxicación media alta en el continuo usado en esta investigación.

Considerando las características de esta diferencia individual, la hipótesis original era que quienes presentaran niveles elevados de ansiedad consumirían alcohol buscando alcanzar los niveles de intoxicación necesarios para enfrentar la incertidumbre y la preocupación ocasionadas por las interacciones sociales y otros estresores presentes en las fiestas. Esta hipótesis se sostiene parcialmente en cuanto que las diferencias más notables con quienes reportan niveles bajos de ansiedad se encuentran en el indicador de dificultad para hablar y se observan diferencias mínimas en el de mareos. Esto mostraría que quienes tienen altos niveles de ansiedad buscan alcanzar niveles de intoxicación alcohólica en las fiestas que les permitan vencer su predisposición a preocuparse por lo que pudiera salir mal.

Sin embargo, esta explicación deja fuera el hecho de que también muestran una mayor predisposición a alcanzar niveles de intoxicación más altos que los llevan incluso a perder la conciencia. Una explicación complementaria, congruente con la concepción teórica de estos constructos (Costa \& Widieger, 1994) y con las correlaciones encontradas entre ansiedad y depresión, es que los participantes con niveles altos de ansiedad a menudo muestran también índices altos de depresión. Probablemente, ambos patrones de consumo se presentan en diferentes momentos en la misma persona, de acuerdo con su estado de ánimo y la diferencia individual dominante en el momento.

Con respecto a la sociabilidad y en contra de nuestras hipótesis, una alta necesidad de convivencia social no deriva, necesariamente, en una mayor exposición a fiestas u otros espacios donde se consumen bebidas alcohólicas. Así, niveles altos de esta diferencia indivi- 
dual no constituyen un factor de riesgo para un consumo excesivo entre los jóvenes.

La variable de búsqueda de sensaciones mostró los efectos esperados sobre el consumo de bebidas alcohólicas. En particular, se asocia a niveles de intoxicación alcohólica que permiten experimentar un estado alterado de conciencia, pero no con indicadores como pérdida de la conciencia, puesto que este estado no permite experimentar sensaciones intensas.

Estos patrones en el consumo de alcohol relacionados con diferencias individuales son congruentes con resultados obtenidos en estudios de neuroimagen. Al utilizar un paradigma para analizar la tolerancia a la demora de recompensas monetarias, se observó que

\section{REFERENCIAS}

Albarracín, D., Blair, T. J., Mark, P. \& Zanna, M. P. (Eds.). (2005). The handbook of attitudes. Mahwah, NJ: Lawrence Erlbaum.

Camacho, A. I. (2005). Consumo de alcohol en universitarios: Relación funcional con los factores sociodemográficos, las expectativas y la ansiedad social. Acta Colombiana Psicología, 8(1), 91-119.

Casanova, L., Borges, G., Mondragón, L., Medina-Mora, M. E. \& Cherpitel, C. (2001). El alcohol como factor de riesgo en accidentes vehiculares y peatonales. Salud Mental, 24(5), 3-11.

Costa, P.T. Jr. \& Widieger, T.A. (1994). Personality disorders and the five factor model of personality. Washington, D.C.: American Psychologist Association.

Diulio, A. R., Silvestri, M. M. \& Correia, C. J. (2014). The role of personality variables in drinking game participation. Addictive Behaviors, 39(7), 1159-1162. doi:10.1016/j.addbeh.2014.02.005

Dubowsky, K. M. (1977). Manual for analysis of ethanol in biological liquids. Reporte No. DOT-TSC-NHTSA-76-4 (HS 802 208), Washington, D.C.: U.S. Department of transportation

Echeburúa, E. (2001). Abuso de alcohol. Madrid: Síntesis. Freitas-Ferrari, M. C., Hallak, J. E. C., Trzesniak, C., Filho, A. S., Machado-de-Sousa, J. P., Chagas, M. H. N., Nardi, A. E. \& Crippa, J. A. (2010). Neuroimaging in social anxiety disorder: A systematic review of the quienes reportan índices más altos de consumo, pero no de abuso de bebidas alcohólicas, muestran un nivel de activación más alto ante la expectativa de posibles pérdidas en el tálamo (Joseph et al., 2015), estructura implicada en los circuitos neurales asociados a estados de ansiedad, y ansiedad social en particular (FreitasFerrari et al., 2010). Ese resultado coincide con el presentado en este estudio, donde personas más ansiosas consumen con mayor frecuencia cantidades moderadas, pero no excesivas de alcohol. Considerando las motivaciones asociadas a diferencias individuales pueden implementarse mejores estrategias preventivas y correctivas de intervención. •

literature. Progress in Neuro-Psychopharmacology \& Biological Psychiatry, 34(4), 565-580. doi:10.1016/j. pnpbp.2010.02.028

Funder, D. C. (2001). Personality. Annual Review of Psychology. 52, 197-221. doi: 10.1146/annurev. psych.52.1.197

Ham, L.S., Debra, A. \& Hope, D. A. (2003). Alcohol and anxiety: Subtle and obvious attributes of abuse in adults with social anxiety disorder and panic disorder. Depress Anxiety, 18(3), 128-139. doi: 10.1002/ da. 10130

Ham, L.S., Hope, D.A., White, C.S. \& Rivers, P.C. (2002). Alcohol expectancies and drinking behavior in adults with social anxiety disorder an dysthymia. Cognitive Therapy and Research, 26(2), 275-288. doi: 10.1023/A:1014582005745

Hull, J.G. \& Young, R.D. (1983). Self-consciousness, self-esteem, and success-failure as determinants of alcohol consumption in male social drinkers. Journal of Personality and Social Psychology, 44(6), 1097 1109. doi.org/10.1037/0022-3514.44.6.1097

Johnson, T.J. \& Cropsey, K.L. (2000). Sensation seeking and drinking game participation in heavy-drinking college students. Addictive Behaviors, 25(1):109-116. doi:10.1016/S0306-4603(98)00118-X

Joseph, J. E., Zhu, X., Corbly, C. R., DeSantis, S., Lee, D. C., Baik, G., Keiser, S., Jiang, Y., Lynam, D. R. \& Kelly, T. H. (2015). Influence of neurobehavioral incentive 
valence and magnitude on alcohol drinking behavior. Neuroimage, 104(1), 373-385. doi:10.1016/j. neuroimage.2014.09.037

Lukassen, J. \& Beaudet, M.P. (2005). Alcohol dependence and depression among heavy drinkers in Canada. Social Science and Medicine, 61(8), 1658-1667. doi:10.1016/j.socscimed.2005.03.019

Martin, C.A., Kelly, T.H., Thomas, H., Rayens, M.K., Brogli, B.R., Brenzel, A., Smith, W.J. \& Omar, H.A. (2002). Sensation seeking, puberty, and nicotine, alcohol, and marijuana use in adolescence. Journal of the American Academy of Child and Adolescent Psychiatry, 41(12), 1495-1502. doi: http://dx.doi. org/10.1097/00004583-200212000-00022

McCambridge, J., Mc Alaney, J. \& Rowe, R. (2011). Adult consequences of late adolescent alcohol consumption: a systematic review of cohort studies. PLoS Medicine, 8(2), 1-13. doi:10.1371/journal. pmed. 1000413

McCrae, R.R. \& Costa, P.T. Jr. (1990). Personality in adulthood. New York: Guilford Press.

McCrae, R.R. \& Costa, P.T. Jr. (1997). Personality trait structure as a human universal. The American Psychologist, 52(5), 509-516. doi: http://dx.doi. org/10.1037/0003-066X.52.5.509

Orozco, R., Borges, G., Mondragón, L. \& Monroy-Nasr, Z. (2005). El lugar donde ocurren las lesiones y su relación con el uso de alcohol. Estudio en sala de urgencias. Salud Mental, 28(5), 50-56.

Medina-Mora, M .E., Villatoro-Velázquez, J.A., FleizBautista, C., Téllez-Rojo, M. M., Mendoza-Alvarado, L. R., Romero-Martínez, M., Gutiérrez-Reyes, J. P., Castro-Tinoco, M., Hernández-Ávila, M., TenaTamayo, C., Alvear-Sevilla, C. \& Guisa-Cruz, V. (2012). Encuesta Nacional de Adicciones 2011. México: INPRFM.

Pluddemann, A., Theron, W.H. \& Steel, H.R. (1999). The relationship between adolescent alcohol use and self-consciousness. Journal of Alcohol and Drug Education, 44(3), 10-20.
Pulido, R., Arras, M., Beauroyre, Y., Cano, L., Coss, León, P., Romo, D., Vázquez J. \& Villafuerte, D. (2002). Consumo de drogas y alcohol en estudiantes de licenciatura de dos universidades particulares de la Ciudad de México. Psicología Iberoamericana, 10(2), 33-41.

Secretaría de Salud (2005). Estadísticas de mortalidad en México: muertes registradas en el año 2003. Salud Pública de México, 47(2), 171-187.

Villatoro, J., Gutiérrez, M., Quiroz, N., Moreno, M., Gaytán, L., Gaytán, F., Amador N. \& MedinaMora, M. E. (2007). Encuesta de consumo de drogas en estudiantes 2006. México: INPRFM . Recuperado de: http://www.uade.inpsiquiatria.edu. $\mathrm{mx} /$ pagina_contenidos/investigaciones/estudiantes_ df/2006/inicio.htm

Villatoro, J., Medina-Mora, M. E., Amador, N., Bermúdez, P., Hernández, H., Fleiz, C., Gutiérrez, M. \& Ramos, A. (2004). Consumo de drogas, alcohol y tabaco en estudiantes del D.F.: medición otoño 2003. Reporte Global. México: InP-SEP. Recuperado de: http:// www.uade.inpsiquiatria.edu.mx/pagina_contenidos/investigaciones/estudiantes_df/2006/cd_2003/ Estudiantes/Encuesta\%202003/Globales/Global\%20 Resultados.htm

Villatoro, J., Medina-Mora, M. E., Rojano, C., Fleiz, C., Villa, G., Jasso, A., Alcántar, M. I., Bermúdez, P., Castro, P. \& Blanco, J. (2001). Consumo de drogas, alcohol y tabaco en estudiantes del Distrito Federal: medición otoño 2000. Reporte Global del Distrito Federal. México: INP-SEP. Recuperado de: http://www.uade. inpsiquiatria.edu.mx/pagina_contenidos/investigaciones/estudiantes_df/cd_2003/Estudiantes/ Encuesta\%202000/REPORTE/Global/TabCont.htm Villatoro, J., Moreno, M., Oliva, N., Freegoso, D., Bustos, M., Fleiz, C., Mujica, R., Mendoza, M.A., López, M.A. \& Medina-Mora, M.E. (2013). Consumo de alcohol, tabaco y otras drogas en la Ciudad de México. Medición 2012. México: InPRFM. Instituto para la Atención y la Prevención de las Adicciones, Administración Federal de los Servicios Educativos para el Distrito Federal. 